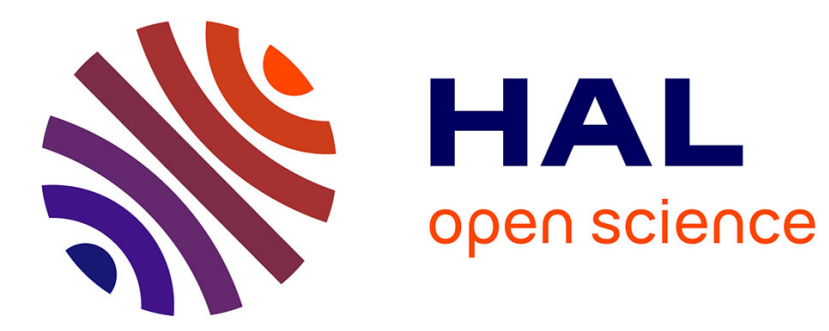

\title{
Novel in situ measurement methods of the total heat transfer coefficient on building walls
}

Adrien François, Laurent Ibos, Vincent Feuillet, Johann Meulemans

\section{To cite this version:}

Adrien François, Laurent Ibos, Vincent Feuillet, Johann Meulemans. Novel in situ measurement methods of the total heat transfer coefficient on building walls. Energy and Buildings, 2020, 219, pp.110004. 10.1016/j.enbuild.2020.110004 . hal-03146489

\author{
HAL Id: hal-03146489 \\ https://hal.science/hal-03146489
}

Submitted on 19 Feb 2021

HAL is a multi-disciplinary open access archive for the deposit and dissemination of scientific research documents, whether they are published or not. The documents may come from teaching and research institutions in France or abroad, or from public or private research centers.
L'archive ouverte pluridisciplinaire HAL, est destinée au dépôt et à la diffusion de documents scientifiques de niveau recherche, publiés ou non, émanant des établissements d'enseignement et de recherche français ou étrangers, des laboratoires publics ou privés. 


\title{
Novel in situ measurement methods of the total heat transfer coefficient on building walls
}

\author{
Adrien François*1,2, Laurent Ibos $^{1}$, Vincent Feuillet ${ }^{1}$, and Johann Meulemans ${ }^{2}$ \\ ${ }^{1}$ Université Paris-Est Créteil, CERTES, F-94010 Créteil, France \\ ${ }^{2}$ Saint-Gobain Research Paris, 39 quai Lucien Lefranc, F-93303 Aubervilliers, France
}

April 16, 2020

\begin{abstract}
The performance gap between the design and the as-built energy performance of buildings is considered a significant risk that may compromise the efforts on meeting the carbon reduction targets set by governments in Europe. The relative importance of irregularities or defects within building walls is more prominent when the thermal insulation level increases. It is thus necessary to quantify the global thermal insulation level of building walls (i.e., including thermal bridges). Classical contact measurement methods only allow local measurements and would thus require the use of a high number of sensors which would limit their applicability. Besides, the presence of thermal bridges prevents the use of contact heat flux measurements because of the 2D or 3D nature of the associated heat transfers through the building walls. To overcome these limitations, the present study proposes an alternative approach consisting in measuring the heat flux on one location and extrapolating its value on the whole building wall through the measurement of the total heat transfer coefficient. Therefore, we developed and/or further improved five different in situ measurement methods of the total heat transfer coefficient on building walls. The measurement methods were thoroughly tested indoor thanks to lab-scale experiments carried out both in steady-state and transient regimes. The results obtained demonstrated the accuracy and the robustness of the proposed methods, as well as their intrinsic limitations. These in situ methods can be used to assess the heat losses on a whole building wall, including the contribution of thermal bridges.
\end{abstract}

Keywords - heat transfer coefficient; building; in situ; operative temperature; measurement

*corresponding author: adrien.francois@u-pec.fr 


\section{Nomenclature}

\section{Acronyms}

DM Double Measurement

HE Harmonic Excitation

HFM Heat Flux Meter

MRT Mean Radiant Temperature

OT Operative Temperature

TEC ThermoElectric Cooler (Peltier modules)

\section{Greek Symbols}$$
\begin{array}{ll}
\lambda & \text { thermal conductivity } \\
\nu & \text { cinematic viscosity }
\end{array}
$$$$
\sigma \quad \text { Stefan-Boltzmann constant }
$$$$
\varphi \quad \text { heat flux }
$$$$
\begin{array}{r}
W . m^{-1} \cdot K^{-1} \\
m^{2} \cdot s^{-1} \\
W \cdot m^{-2} \cdot K^{-4} \\
W \cdot m^{-2}
\end{array}
$$

\section{Roman Symbols}

A amplitude

a thermal diffusivity

$f \quad$ frequency

FT Fourier Transform

$h \quad$ global heat transfer coefficient

$N \quad$ number of points

$T \quad$ temperature

$u \quad$ uncertainty

Gr Grashof number

$\mathrm{Nu} \quad$ Nusselt number

Ra Rayleigh number

\section{Superscripts}

$\sim \quad$ complex harmonic

app apparent

\section{Subscripts}

c convective

op operative

$\mathrm{r}$ radiative

\section{Introduction}

The domestic sector plays a major role in greenhouse gas emissions. In order to reduce the primary energy consumption and minimize the impact of climate change, significant efforts have to be made on building thermal insulation. The performance of building fabrics is usually calculated but seldomly measured. On this matter, many authors observed significant differences between in situ measurements and predictions: this is the "performance gap" [1]. The experimental quantification of building envelope performances is not straightforward. One prerequisite for energy losses quantification is the measurement of heat fluxes on building walls. The heat transfers are usually not one dimensional and the losses are not uniform all over the walls. For instance, the presence of irregularities in the envelope (usually referred as thermal bridges) locally generate additional heat losses. In steady-state, heat flux measurements can be related to a wall thermal resistance. In transient regimes, they can also pro$K$ vide valuable insights on the internal structure of the wall.

At the present time, to the best of the authors' knowledge, there is a lack of measurement standardized method for performing an in situ quantitative diagnosis of thermal insulation of building walls. Several methods were developped in the acamedic sphere and a lot of efforts are currently being made to improve their accuracy, rapidity and applicability. Bienvenido-Huertas et al. recently published a comprehensive review [2] summarizing most of these methods. For the sake of completeness, let us add the QUB/e method [3] [4] to this review. However, these methods are not mature enough to be widely used by the community. In contrast, most experimental methods for the measurement of material thermal conductivity are now standardized for instance. For the thermal insulation of building walls, there are only two existing standardized techniques, namely ISO 9869-1 [5] and 9869-2 [6]. Nevertheless, they fail in accurately evaluating a thermal $s^{-1}$ resistance or $U$ transmission coefficient in many situations, especially if heat transfers are far from being in steady-state regime and if the indoor-outdoor temperature gradient is too small. This is mainly because these approaches are passive methods, therefore strongly influenced by climate conditions.

Thus, there is a need for the development of new in situ $K$ diagnosis methods that could be used in most of measurement conditions. The main objective of this study is to propose a procedure able to estimate in situ the heat losses onto a whole wall surface. This method should be able to take into account thermal bridges contributions. As it will be exposed further in this section, the estimation of the wall heat losses will be based on the knowledge of the global heat exchange coefficient $h$.

Consequently, this paper will focus on the in-situ measurement of the heat exchange coefficient on a wall surface and on the determination of the associated uncertainties. Several methods will be investigated. They should be able to take into account both convective and radiative heat exchanges. Their applicability for measurements in both static and transient regimes will be investigated. Finally, measurement devices tested have to be as non-intrusive as possible: they should not modify convective and radiative exchanges. The general methodology proposed is exposed in the following.

Given the small temperature differences encountered in buildings, radiative heat fluxes can be linearized. The heat flux $\varphi$ on a wall surface is then given by:

$$
\varphi=h_{\mathrm{c}}\left(T-T_{\mathrm{air}}\right)+h_{\mathrm{r}}\left(T-T_{\mathrm{MRT}}\right)
$$

with $h_{\mathrm{c}}$ and $h_{\mathrm{r}}$ the convective and radiative heat exchange coefficients, $T_{\mathrm{MRT}}$ the Mean Radiant Temperature and $T_{\text {air }}$ the air temperature outside the near-wall boundary layer. The radiative coefficient is given by:

$$
h_{\mathrm{r}}=4 \epsilon \sigma\left(\frac{T+T_{\mathrm{MRT}}}{2}\right)^{3}
$$


with $\epsilon$ the surface emissivity (grey body assumption) and $\sigma$ the Stefan-Boltzmann constant. The surface heat flux can also be given as a function of the global heat exchange coefficient $h$ and the so-called "Operative temperature" $T_{\text {op }}[6]$$$
\varphi=h\left(T-T_{\mathrm{op}}\right)
$$

with $h=h_{\mathrm{c}}+h_{\mathrm{r}}$ and $T_{\mathrm{op}}=\left(h_{\mathrm{c}} T_{\mathrm{air}}+h_{\mathrm{r}} T_{\mathrm{MRT}}\right) / h$. It is important to point out that both $h$ and $T_{\text {op }}$ are local quantities and might not be uniform all over the considered wall. Heat Flux Meters (HFM) are commonly used to measure the heat flow across a plain wall where the transfers are onedimensional. However, in the presence of thermal bridges, the heat transfers are $2 \mathrm{D}$ or $3 \mathrm{D}$ and HFM cannot be used anymore. More generally, heat losses on a building wall are rarely uniform. A local heat flux measurement cannot be representative of the whole wall. For instance, Fig. 1 presents a thermal image of a wall that includes several thermal bridges (described in [7]). The surface temperature is clearly not homogeneous. Therefore, an accurate quantification of the losses would require the use of many sensors which might be expensive as well as time consuming.

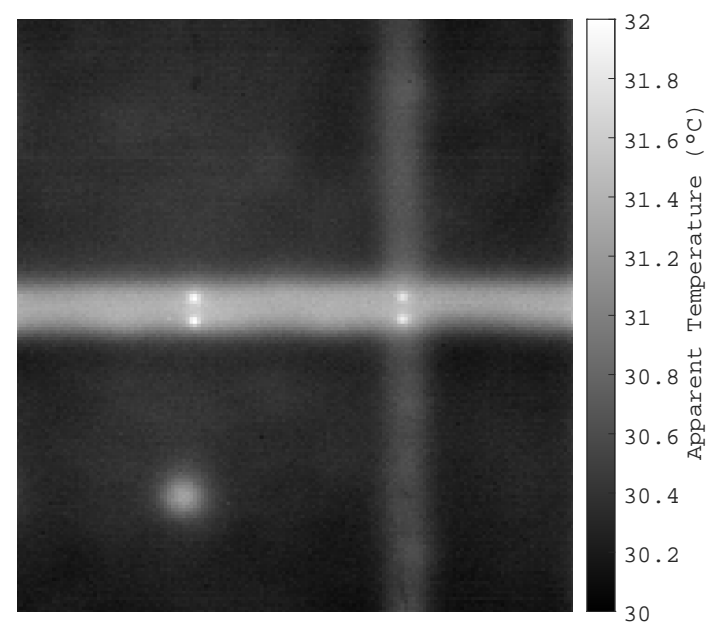

Figure 1: Thermal image of a non-homogeneous wall because of the presence of thermal bridges (from [7])

The proposed solution is to extrapolate the heat flux measured on a sound area 1 to a nearby area 2 . Indeed, if the two areas are close and at a similar altitude, $h$ and $T_{\text {op }}$ may be considered equal on them. It comes:

$$
\varphi_{2}=\varphi_{1}+h\left(T_{2}-T_{1}\right)
$$

Then, by measuring $\varphi_{1}$ with a HFM, $T_{2}-T_{1}$ with thermocouples or infrared thermography, and $h$ with a specific device, the second heat flux $\varphi_{2}$ can be derived. Therefore, this paper focuses on the measurement of $h$ in unsteady conditions in order to extrapolate a local contact measurement to the rest of the wall, even in the presence of thermal bridges. One of the main difficulty is that the operative temperature $T_{\mathrm{op}}$ is not known. It is usually assumed equal to the air temperature. This assumption is acceptable in steady-state indoor conditions in well insulated buildings : the room is globally at thermal equilibrium. It is however less valid in old buildings where cold surfaces due to the lack of insulation generate thermal imbalance. Moreover, this assumption is also false in transient regimes when radiation and convection have a very different behavior. The following paragraphs present several $h$-measurement methods developed and experimented here. A particular attention will be paid to the evaluation of measurement uncertainties.

The next section summarizes the existing methods for $h$ coefficient evaluation in buildings and their limitations. Section 3 presents the experimental setup built and the five $h$ measurement methodologies developed whereas section 4 details the calculation of the measurement uncertainties. Sections 5 and 6 present results obtained in steady and transient states respectively. These results are discussed in section 7 .

\section{Common methods for global $h$ - coefficient evaluation}

A first approach is to use a standard value. ISO standard 6946 [8] defines default $h$-values to use in building numerical simulations: 7.7 and $20 \mathrm{~W} . \mathrm{m}^{-2} \cdot K^{-1}$ for indoor and outdoor vertical surfaces, respectively. This provides an order of magnitude of the heat exchange coefficient but does not take into account the configuration specificity (ventilation rate, surface temperature differences, geometry, etc).

A second approach consists in using one of the many empirical correlations developed in the literature to estimate the convective heat transfer coefficient $h_{\mathrm{c}}$ [9]. The radiative component $h_{\mathrm{r}}$ is then estimated from surface temperature and emissivity measurements. However, most commonly used correlations are based upon experiments using small free edged heated plates under natural convection [10]. This is usually not representative of in situ conditions in buildings. Even when the conditions are similar, there is an important discrepancy between correlations. For instance, Dascalaki [11] compared fifty-eight $h_{\mathrm{c}}$ correlations (see [9] for instance). Only ten of them were for enclosures, with a variability of almost $50 \%$ in Nusselt number. The latter is a dimensionless number that characterizes heat transfers on a surface :

$$
N u=\frac{h_{\mathrm{c}} L_{\mathrm{c}}}{\lambda}
$$

with $L_{\mathrm{c}}$ the characteristic dimension of the problem, $\lambda$ the air thermal conductivity.

Most correlations link the Nusselt number with the Prandtl number $\operatorname{Pr}$ as well as either the Grashof $G r$ or the Rayleigh $R a$ number :

$$
\operatorname{Pr}=\frac{\nu}{a}
$$

with $\nu$ and $a$ the air cinematic viscosity and thermal diffusivity.

$$
G r=\frac{g \beta \Delta T L_{c}^{3}}{\nu^{2}}
$$

with $g$ the gravitational acceleration, $\beta$ the air thermal expansion coefficient and $\Delta T$ the air-to-wall temperature difference.

$$
R a=G r \times P r
$$


In a building application, the calculation of $G r$ (or similarly $R a)$ is not straightforward at all. Indeed, because the temperature is usually not uniform in a room, the choice of the location where the air temperature is measured has a major impact on $\Delta T$ and therefore on $G r$. Consequently, an empirical correlation may not able to provide an accurate estimation of the local heat transfer coefficient.

Third, one of the best ways to have a proper estimation of the $h$-coefficient is probably to measure it. Almost every measurement method found in the literature focuses on the convective component $h_{\mathrm{c}}$. In some cases, substituting low emissivity surfaces with high emissivity ones would enable to include the radiative exchanges in the measured quantity.

On the one hand, for outdoor applications, the measurement methods are based on the use of heated plates. In Jayamaha's work [12], a measuring device is placed in a cutout of a plate. It is made out of a HFM of low emissivity mounted on a film heater itself fixed on an insulating material. The film is fed so that the plate temperature is kept constant. The $h_{\mathrm{c}}$-coefficient is derived from a thermal balance including the surface heat flux, surface-to-air temperature difference and radiative exchanges. The temperature difference spans from 12 to $20 \mathrm{~K}$. Many other authors similarly performed outdoor $h_{c}$ measurements. Their device is not always fixed in a cut-out of a plate but is also based on a heating component and a heat flux measurement. Hagishima [13] generated $h_{\mathrm{c}}$ maps on building canopy surfaces. Loveday [14] focused on small buildings. Ito [15] proposed a slightly different method as he used two distinct plates heated at different temperatures. By working with the heat flux and temperature differences between them, the measurement of the air temperature is no longer needed. Ohlsson [16] made a thorough uncertainty estimation of the methods developed by Ito and Loveday. This work is based on lab-scale measurements of small plate under forced convection. In another study [17], the same authors investigated the impact of response time of usual outdoor $h$-measurement devices. They suggest modifications to reduce this response time in order to be able to capture faster variations of $h$.

On the other hand, in indoor applications, every measurement method was made in steady-state and most of them in a controlled environment. Khalifa [18] made $h_{\mathrm{c}}$ measurements in a $3 \times 2.4 \times 2 \mathrm{~m}^{3}$ test-cell. The study covers most widely used heating configurations in buildings. Radiative heat transfers are neglected. Awbi [10] used a similar protocol in a $4 \times 3 \times 2.5 \mathrm{~m}^{3}$ chamber. The main difference is that heating plates were used to provide surface heating on each wall. Wallenten [19] measured the $h_{\mathrm{c}}$ in an environment more realistic of building situations: a $3 \times 3.6 \times 2.4 \mathrm{~m}^{3}$ room equipped with radiator and ventilation system. The $h_{\mathrm{r}}$ value is calculated from temperature measurements and view factors. These methods are not applicable in situ. Indeed, the heat fluxes were derived from temperature measurements on each side of thin, non-insulated walls of perfectly known conductivity: the wall forms a HFM itself. In addition, the $h$-coefficient was calculated taking a space average of the air temperature as reference temperature. According to Wallenten [19], this reference temperature may be arbitrary chosen for numerical simulation purposes. In our applications however, the reference temperature has to be the operative temperature. These methods may be extended to the measurement of the total $h$-coefficient by swapping low emissivity surfaces by high emissivity ones.

An alternative for indoor $h_{\mathrm{c}}$ measurement is the so-called "Mayer Ladder". This concept can be traced back to Mayer [20] and has the advantage of being applicable in situ. It is based on the measurement of the air boundary layer thickness close to the studied surface. The Mayer ladder was not originally developed for building applications, and was designed for a temperature difference $\Delta T$ of $22 \mathrm{~K}$. Delaforce [21] extended the method to the building sector, with $\Delta T$ around 2 K. Works of Irving [22], Griffith [23] and Davis [24] also involved the use a of Mayer Ladder in buildings. Nevertheless, unlike previously presented methods, the Mayer ladder cannot be modified to include radiative heat transfers for the direct measurement of the total $h$-coefficient. The radiative component would have to be estimated from temperature and emissivity measurements of the surrounding environment.

The only reference found that deals with a direct measurement of the total $h$ coefficient is the ISO 9869-2 standard [6]. It is derived from Kato [25] and proposes a measurement device. It is made out of a HFM sandwiched between a copper plate of high emissivity on the front side and a heating resistance on the rear side. The latter is insulated with a piece of polystyrene and the complete device placed in front of the wall. Thanks to the heating resistance, the device-to-air temperature difference is kept between 3 and $10 \mathrm{~K}$. The operative temperature is measured by another device using a thermocouple inserted between a copper plate of high emissivity and some polystyrene. This device is referred as "ET sensor", for Environment Temperature sensor. It is said that the device should be placed near the wall but should not touch it either. The initial method proposed by Kato [25] used a globe sensor, but the exchanges around a sphere are not representative of those on a wall surface. This method is unfortunately only applicable in steady-state. In addition, because of the high temperature elevation on the $h$-measurement device, the natural convection conditions on its surface must be different from the ones on the wall surface. This probably introduces a measurement bias.

Consequently, the literature review showed very few in situ measurement methods of the global heat exchange coefficient $h$ on a building wall. This paper presents five different measurement methods of indoor $h$-coefficient. Three of them are original methods. The other two are derived from Ito [15] and the ISO 9869-2 standard [6] respectively. They are also applicable on outdoor surfaces.

\section{Methodology and setup}

\subsection{Experimental setup}

An experimental $1.2 \times 2 \mathrm{~m}^{2}$ wall was built in laboratory to test the methods developed. It consists of a first gypsum layer (13 mm thick) behind which two different materials $(90 \mathrm{~mm}$ thick) are disposed: expanded polystyrene on one side and cellular concrete on the other side. The objective was to have two different zones with heat transfers which can be considered 1D. The heat flux on the second zone will be extrapolated from contact heat flux measurement on the first zone. Because the second zone is also $1 \mathrm{D}$, a contact measurement on it allows to validate the methodology. The setup is fixed on an internal wall of a $4 \times 5 \times 3 \mathrm{~m}^{3}$ room. An insulating frame 
around the setup was added to limit lateral losses. Several $h$ measurement devices are attached to this wall. They are presented in details in the next section. Fig. 2 illustrates the layout of the experimental wall and shows the location of the $h$ measurement devices. All the devices are based on contact measurements and are labeled by letters the significations of which are detailed below. Fig. 3 shows a picture of the instrumented wall.

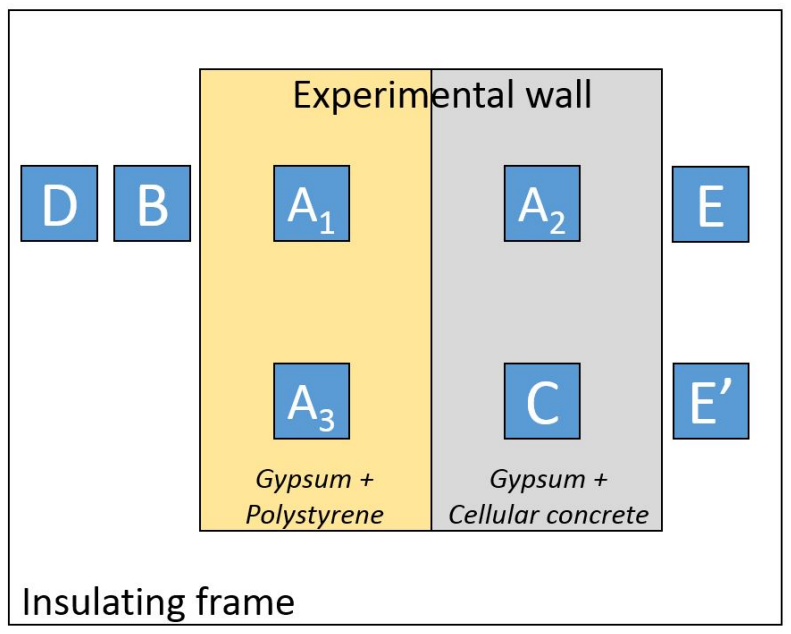

Figure 2: Schematic front view of the studied experimental wall within its insulating frame and location of all the devices used (labeled by letters)

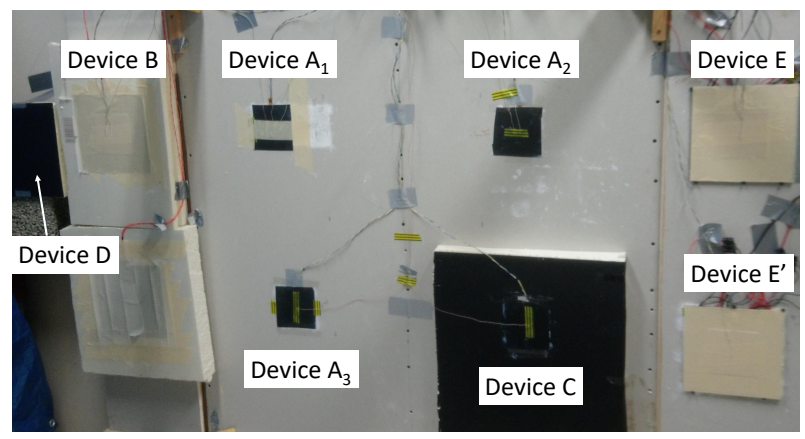

Figure 3: Picture of the instrumented setup

Heat fluxes are measured thanks to $100 \times 100 \mathrm{~mm}^{2}$ heat flux meters from the Captec manufacturer. They have a $0.54 \mathrm{~mm}$ thickness and a sensitivity superior to $60 \mu \mathrm{V} .\left(\mathrm{W} . \mathrm{m}^{-2}\right)^{-1}$ The sensitivities uncertainty is of $3 \%$. Temperatures are measured with 2 mm-thick type-T thermocouples. The latter were calibrated with a platinum sensor over the range $0^{\circ} \mathrm{C}$ - $50^{\circ} \mathrm{C}$. The reference platinum sensor was used with an AOIP TM6612 temperature datalogger. The measurement uncertainty of this reference is of $0.2^{\circ} \mathrm{C}$. An external calibration of these devices was performed in order to ensure the metrological traceability to the ITS-90 [26]. HFM are covered with sheets of paper having the same infrared emissivity than the studied wall: 0.94 . This value is the spectral average over the long-wave infrared band ( 8 to $14 \mu \mathrm{m})$. The emissivities were measured with an infrared spectrometer (Fron- tier model, from Perkin-Elmer) equipped with an integrating sphere (from Pike). A diffusing gold surface (SpectraGold) was used as reference and its reflectance was measured by an independent method at France's National Metrology Institute (NMI)

In most configurations, only temperature differences between two areas are required rather than absolute temperatures. Therefore, each temperature difference is directly measured by fixing one junction of a thermocouple on the first area, and the second junction on the other area. There is therefore no cold junction compensation and the measured quantity is directly the temperature difference between the two junctions. This reduces the measurement uncertainties. The measured voltage is converted into Kelvin degrees thanks to the NIST polynomial [26]. In addition, the air temperature is measured $15 \mathrm{~cm}$ from the wall by a thermocouple shielded from radiation with aluminum tape. To generate unsteady configurations, four $500 \mathrm{~W}$ electric fan heaters are arranged into the room. Temperatures and heat fluxes are recorded every $3 \mathrm{~s}$ and averaged every 5 points. HFMs and TCs are plugged on National Instruments NI9214 conditioning modules. These modules communicate with a PC computer via a 8-slot NI CompactDAQ USB chassis. The experimental setup is monitored by a LabView application.

It may be noted that the different sensors used (thermocouples and HFMs covered with paper) are supposed nonintrusive. This hypothesis is sensible given their negligible thickness and thermal resistance when compared to the ones of the wall.

\subsection{Double measurement method 1 (DM1)}

This method and the following one rely on measurements on two different surfaces (noted $A_{1}$ and B in Fig. 2). It is based on the same principle as developed by Ito [15]. First, a HFM is placed on the wall. This is the device A shown in Fig. 4. Let $\varphi_{A}$ and $T_{A}$ be the heat flux and temperature measured on its surface. A heating resistance is placed next to it and another HFM on top of it measures $\varphi_{B}$ and $T_{B}$ (see device $\mathrm{B}$ in Fig. 5). The resistance is fed with a constant power to ensure that $T_{B}$ is always about $1^{\circ} \mathrm{C}$ higher than $T_{A}$. A lower value would reduce the signal to noise ratio while a too high temperature difference would significantly alter the convection pattern near the wall and introduce a bias in the measurement.

The heat flux on each surface is given by Eq. 3. Both $h$ and $T_{\mathrm{op}}$ are then supposed equal on the two surfaces. The heat transfer coefficient is therefore expressed as:

$$
h=\frac{\varphi_{B}-\varphi_{A}}{T_{B}-T_{A}}
$$

This way, $h$ can be determined without knowledge of $T_{\text {op }}$. This method has the advantage of being applicable in steady state as well as in transient regimes. The heating resistance ensures that $T_{B}-T_{A}>0$. 


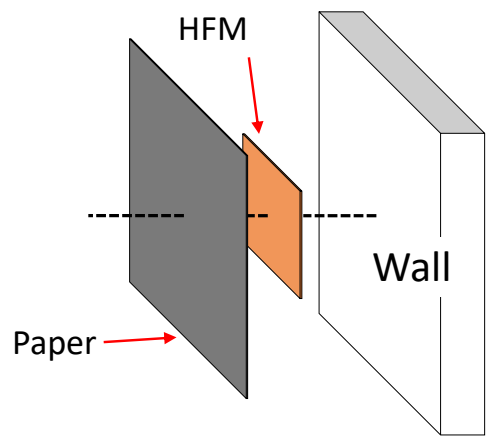

Figure 4: Device A: simple HFM directly fixed on the wall (used in DM1 and DM2 methods)

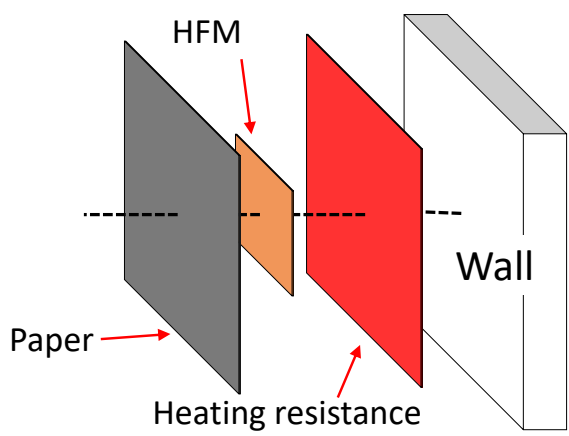

Figure 5: Device B: a HFM mounted on a heating resistance fixed on the wall (used in the DM1, OT1 and OT2 methods)

\subsection{Double measurement method 2 (DM2)}

This method, developed by the authors, is a variation of the previous one. It uses devices $\mathrm{A}_{3}$ and $\mathrm{C}$ of Fig. $2\left(\mathrm{~A}_{3}\right.$ is preferred to $\mathrm{A}_{1}$ because it is closer to $\mathrm{C}$ and at the same altitude). The installation is similar to the previous one, only the heating resistance is replaced by a piece of material. Device C is illustrated in Fig. 6. The thermal effusivity of this material should be different from that of the wall. A $50 \mathrm{~mm}$ thick piece of extruded polystyrene was used here. Basically, a change in the internal temperature of the room induces a temperature difference between surfaces $\mathrm{A}$ and $\mathrm{C}$ because they are mounted on different materials. Similarly to Eq. 9, it comes:

$$
\varphi_{C}-\varphi_{A}=h\left(T_{C}-T_{A}\right)
$$

with $\varphi_{C}$ and $T_{C}$ the heat flux and the temperature measured on the added material, respectively. The calculation of $h$ is not as straightforward as in the previous method because there may be moments when the temperature difference $T_{C}-T_{A}$ is null. However, plotting $\varphi_{C}-\varphi_{A}$ as a function of $T_{C}-T_{A}$ gives a straight line of slope $h$. This method has the advantage of being very simple to implement since it only requires a piece of polystyrene and two HFM. However, an external heat source is needed so this method is not applicable in steady-state. In the present study, the room air temperature is heated with electric fan heaters. For outdoor applications, the thermal load could come from solar radiation or from daily variations in the air temperature.

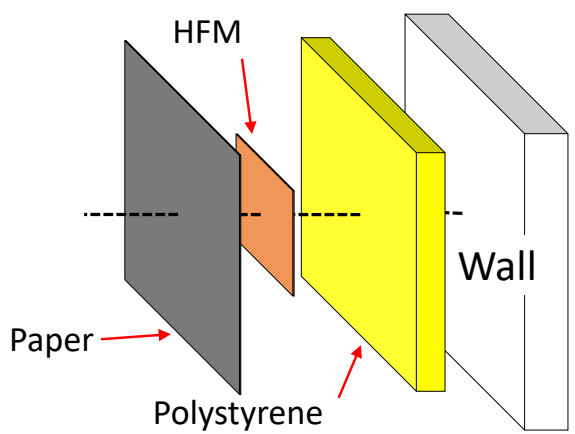

Figure 6: Device C: a HFM mounted on a piece of extruded polystyrene fixed on the wall (used in the DM2 method)

\subsection{Operative temperature measurement method 1 (OT1)}

This method as well as the next one rely on the measurement of the operative temperature $T_{\mathrm{op}}$ on the wall surface. It is based on the ISO 9869-2 standard [6]. The operative temperature is measured thanks to a thermocouple sandwiched between a copper plate and a piece of polystyrene, as presented in Fig. 7 (Device D). According to [6], the device should be placed near the wall but should not touch it. The copper plate ensures the surface temperature uniformity. Thanks to the insulating material on the rear side, the copper plate mainly exchanges with the surrounding environment both by convection and radiation. At equilibrium, by definition of $T_{\mathrm{op}}$ :

$$
T_{D}=T_{\mathrm{op}}
$$

Then, Eq. 3 is used to derive $h$ from the heat flux on the heated surface (Device B):

$$
h=\frac{\varphi_{B}}{T_{B}-T_{\mathrm{op}}}
$$

Similarly to the DM2 method, the main advantage of this method is its simplicity. Unfortunately, it requires that the copper plate be at thermal equilibrium with the surrounding environment. This assumption is usually valid in steady state. It is however less valid when the environment temperature conditions change by a few degrees is one hour, such as in our experiment in which the air is heated.

In addition, there is no guarantee in transient regimes that $T_{B}-T_{\mathrm{op}} \neq 0$ during the total duration of the experiment. Therefore, a linear regression is sometimes preferable than the division of Eq. 12. 


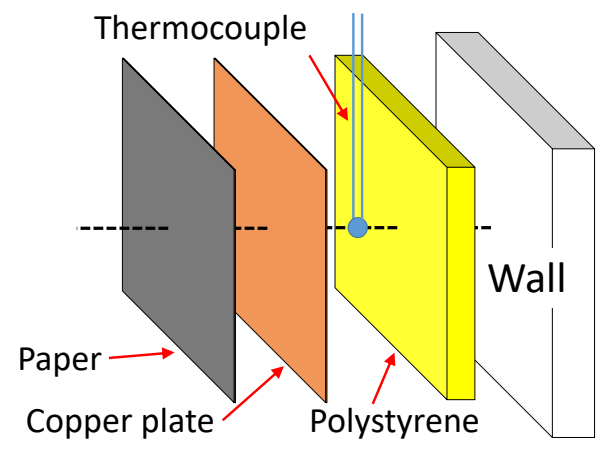

Figure 7: Device D: a thermocouple sandwiched between a copper plate and extruded polystyrene (used in the OT1 method)

\subsection{Operative temperature measurement method 2 (OT2)}

This method is a variation of OT1 method and was developed by the authors. The working principle is similar, but the way $T_{\mathrm{op}}$ is measured is different: Device E (see Fig. 8) is used instead of Device D. Essentially, an active system allows to compensate non equilibrium conditions when the environment temperature varies rapidly. As illustrated in Fig. 8, a matrix of nine "Thermoelectric Coolers" (TEC, also referred as "Peltier modules") is sandwiched between two $200 \times 200 \mathrm{~mm}^{2}$ aluminum plates of $1 \mathrm{~mm}$ thickness. The later are implemented to make the temperature uniform either side of the device. This assembly, which is around $5-\mathrm{mm}$ thick, is fixed on the wall. A $100 \times 100 \mathrm{~mm}^{2}$ heat flux meter is placed on the front side. In practice, the feeding of the Peltier modules is live-controlled so that the surface heat flux $\varphi_{E}$ is always null. If there is no heat flux on the surface of the device, it means that its temperature $T_{E}$ is equal to the operative temperature $T_{\mathrm{op}}$ :

$$
T_{E}=T_{\mathrm{op}}
$$

Therefore, $T_{\mathrm{op}}$ is directly given by the measurement of the device surface temperature. From this, Eq. 12 is used to derive the $h$ coefficient.

The voltage command $U$ of the TECs is updated at every time step so that $\varphi_{E}$ remains close to zero. The use of a PID controller was first considered. Unfortunately, the considered system taking $U$ as input and $\varphi_{E}$ as output is not invariant in time. Indeed, depending on the history of the system, its response will differ: if the temperature of the rear side changes, the required voltage to maintain $\varphi_{E}=0$ changes as well. Consequently, classical control systems are not applicable. Therefore, a simple iterative approach was retained. At every time step $t_{i}, \varphi_{E}$ is measured. If it is too high or too low (a threshold value $\varphi_{\mathrm{lim}}=0.01 \mathrm{~W} \cdot \mathrm{m}^{-2}$ is defined), the command $U$ is incremented or decremented by a step $\delta U$ :

$$
\begin{cases}\varphi_{E}\left(t_{i}\right)<-\varphi_{\mathrm{lim}} & : U\left(t_{i}\right)=U\left(t_{i-1}\right)+\delta U \\ -\varphi_{\mathrm{lim}}<\varphi_{E}\left(t_{i}\right)<\varphi_{\mathrm{lim}} & : U\left(t_{i}\right)=U\left(t_{i-1}\right) \\ \varphi_{E}\left(t_{i}\right)>\varphi_{\mathrm{lim}} & : U\left(t_{i}\right)=U\left(t_{i-1}\right)-\delta U\end{cases}
$$

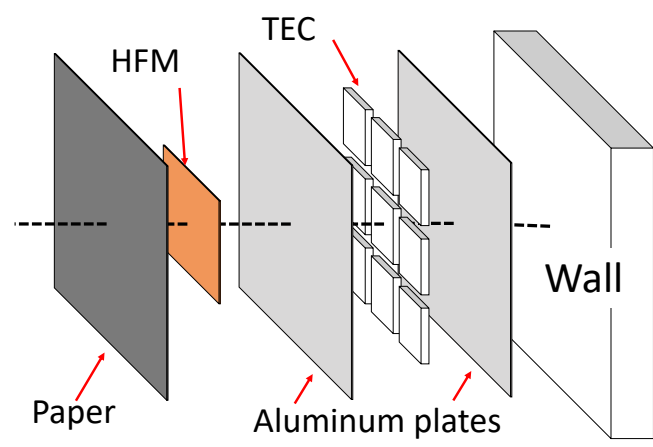

Figure 8: Device E: a HFM mounted on an array of Thermoelectric coolers fixed on the wall (used in the OT2 and HE methods)

\subsection{Harmonic excitation method (HE)}

This last novel method was also developed by the authors. A sinusoidal thermal load is applied to a surface. It uses another Device E (shown in Fig. 8 and referred as device E' in Fig. 2): a HFM mounted on TEC. This time, the thermoelectric coolers are fed with a sinusoidal electric signal so that they deliver a harmonic thermal power.

In the frequency domain, the influence of $T_{\mathrm{op}}$ (supposed constant) disappears:

$$
\widetilde{\varphi}=h \widetilde{T}
$$

where the superscript $\sim$ denotes the complex harmonic notation. The $h$ coefficient is given by :

$$
h=\Re\left(\frac{\widetilde{\varphi}}{\widetilde{T}}\right)=\frac{A_{\varphi}}{A_{\mathrm{T}}} \cos (\zeta)
$$

with $\Re$ the real part operator, $A_{\varphi}$ and $A_{\mathrm{T}}$ the surface heat flux and temperature amplitudes and $\zeta$ the phase lag between the two signals. The $h$ value is derived from the discrete Fourier transforms $F T_{\mathrm{T}}$ and $F T_{\varphi}$ of the signals:

$$
h=\Re\left(\frac{F T_{\varphi}\left(k_{f}\right)}{F T_{\mathrm{T}}\left(k_{f}\right)}\right)
$$

with $k_{f}$ the index of the harmonic corresponding to the excitation frequency $f$.

A preliminary study was carried out to identify the best operating conditions of the device. First, the oscillation frequency $f$ has to be high enough for $T_{\mathrm{op}}$ to be considered constant during several periods but larger than the HFM response time. A period of 5 min proved to be a good optimum. Second, the amplitude of the oscillations cannot be too small (for the signal-to-noise ratio to be high enough) or too large (a significant temperature increase would change the convective pattern of the air flow and introduce a bias on the measurement). Therefore, the power supplied to the TECs was set such that the temperature oscillation amplitude was close to $0.3 \mathrm{~K}$.

\section{Uncertainty calculations}

For each method, the measurement uncertainties are calculated. According to the GUM (Guide to the expression of uncertainty in measurements, [27]), measurement uncertainty 
components should be grouped into two categories: type-A and type-B. Type-A uncertainties are evaluated by statistical methods while type-B ones are evaluated otherwise. For each measurement method, both types of uncertainties are estimated in order to work out the combined uncertainty:

$$
u=\sqrt{u_{\text {type-A }}^{2}+u_{\text {type- }}^{2}}
$$

\subsection{Type-A uncertainty}

The type-A uncertainty is founded on statistical distribution.

First, the DM2 and OT2 methods are based on a linear regression $Y=h \times X+b$ in which the slope is the desired $h$ value. $X$ is a temperature difference while $Y$ is either a heat flux (OT2 method) or a heat flux difference (DM2 method). The statistical uncertainty on the slope obtained by linear regression is commonly given by [28]:

$$
u_{\mathrm{h}}=\frac{1}{\sqrt{N}} \frac{u_{\mathrm{Y}}}{\operatorname{Var}(X)}
$$

With $N$ the number of points, $u_{\mathrm{Y}}$ the standard deviation of the noise on the $Y$ vector and $\operatorname{Var}(X)$ the statistical variance of the $X$ vector. Yet, this common equation supposes that $X$ is perfectly known which is not the case in our application. Indeed, both $Y$ and $X$ are measured quantities. Equation 19 may be modified to include the standard deviation $u_{\mathrm{X}}$ of the noise on $X[28]$ :

$$
u_{\mathrm{h}}=\frac{1}{\sqrt{N}} \frac{\sqrt{u_{\mathrm{Y}}^{2}+h^{2} u_{\mathrm{X}}^{2}}}{\operatorname{Var}(X)}
$$

Second, the HE method is based on Fourier transforms of signals. The calculation of the uncertainty on $h$ in this situation is detailed in appendix A. Calculations lead to :

$$
u_{\mathrm{h}}=\frac{1}{A_{\mathrm{T}}} \sqrt{u_{A_{\varphi}}^{2}+\left(\frac{A_{\varphi}}{A_{\mathrm{T}}}\right)^{2} u_{A_{\mathrm{T}}}^{2}}
$$

with $A_{\mathrm{T}}$ and $A_{\varphi}$ the temperature and heat flux amplitudes, $u_{A_{\mathrm{T}}}$ and $u_{A_{\varphi}}$ their corresponding uncertainties. The amplitudes are worked out from the Fourier transform harmonic corresponding to the excitation frequency:

$$
A_{\mathrm{T}}=\left|F T_{\mathrm{T}}\left(k_{j}\right)\right|
$$

similarly with $\varphi$. The uncertainties are given by the noise on the other harmonics:

$$
u_{A_{\mathrm{T}}}^{2}=\left.\operatorname{Var}\left(F T_{\mathrm{T}}\left(k_{j}\right)\right)\right|_{k \neq k_{j}}
$$

similarly with $\varphi$.

\subsection{Type-B uncertainty}

In this case, type-B uncertainties correspond to systematic errors. Let $\alpha$ be the desired quantity, function of $m$ parameters $\beta_{1}, \ldots, \beta_{m}$. The propagation law [27] relates the uncertainty over $\alpha$ to the ones of the $\beta_{i}$ :

$$
u(\alpha)=\sqrt{\sum_{i=1}^{m}\left(\frac{\partial \alpha}{\partial \beta_{i}} u\left(\beta_{i}\right)\right)^{2}}
$$

According to the HFM manufacturer, heat fluxes are measured with an uncertainty of $3 \%$. In addition, the measured temperature differences are given with an accuracy of $0.03 \mathrm{~K}$. This value originates from the Seebeck coefficients used to convert voltage in temperature [29].

\section{$5 \quad$ Steady-state results}

First, results obtained in pseudo-steady-states are given here (the air heaters are not used). The measurements presented here were made indoor during an afternoon of July 2018 in Créteil, France. The internal air temperature measured close to the wall was almost constant all the way through the experiment : it monotonously decreased from 28.15 to $28^{\circ} \mathrm{C}$. All $h$-measurement devices are implemented simultaneously on the experimental wall. The DM2 method is not used here as it is not applicable in steady-state. Because measurements are made in situ, therefore the temperature inside the room is not exactly constant. Yet, its variations are slow and of small amplitude such that a pseudo-steady-state is reached. A representative sample of the results are given in figures 9 to 12 . The evolution of the measured $h$-value for 7 hours is plotted with uncertainty bars. A coverage factor $\mathrm{k}=2$ was chosen. The mean values are summarized in Tab 1.

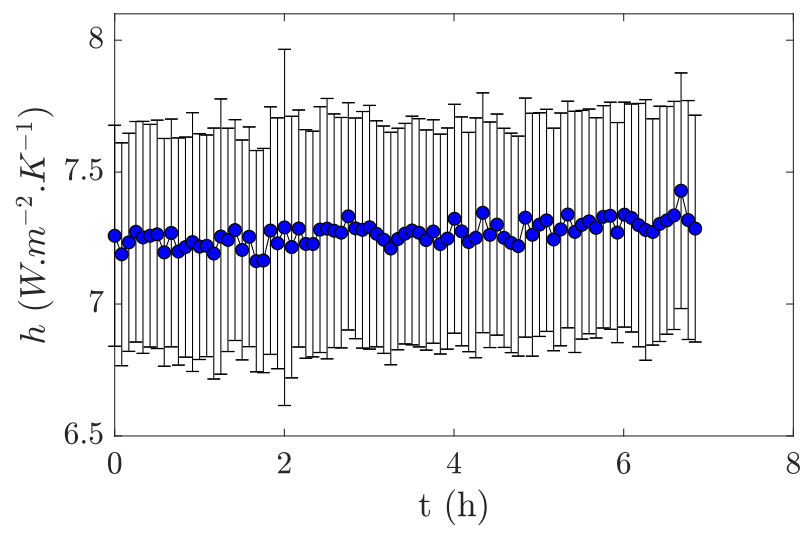

Figure 9: Steady-state $h$ measurement: DM1 method

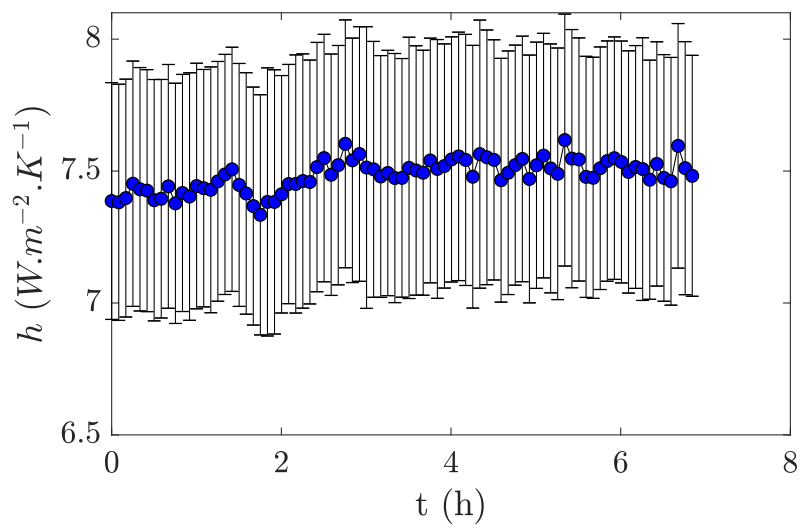

Figure 10: Steady-state $h$ measurement: OT1 method 


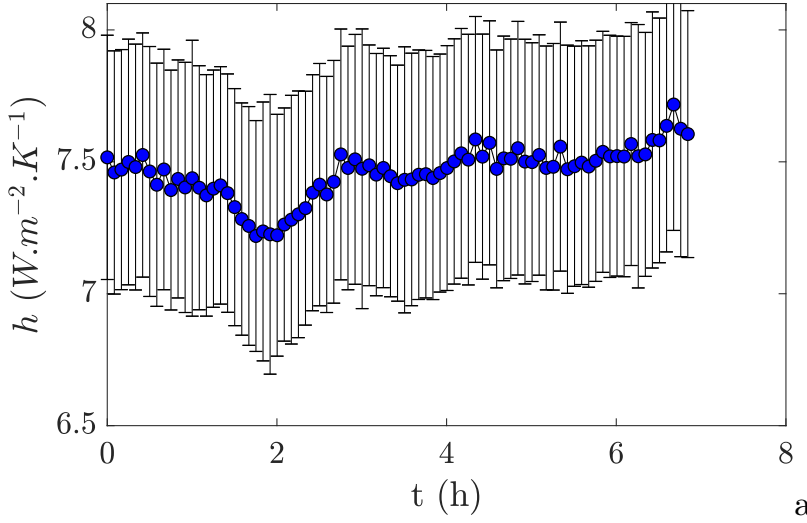

Figure 11: Steady-state $h$ measurement: OT2 method

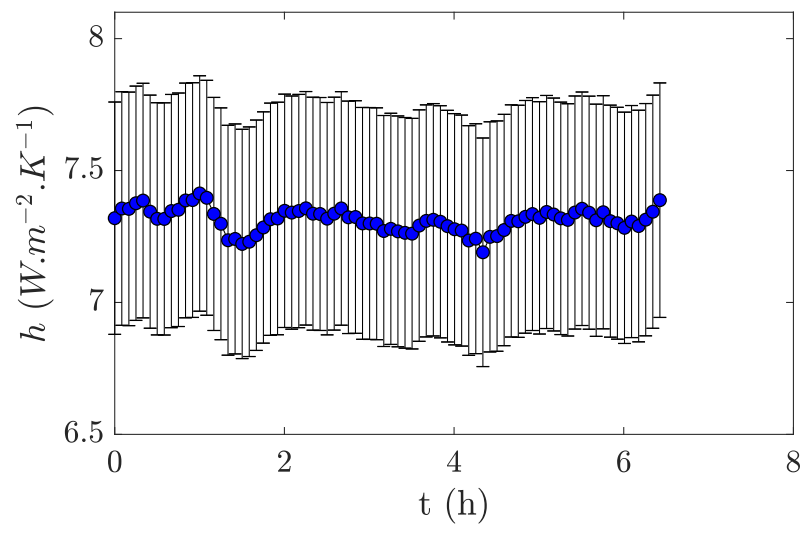

Figure 12: Steady-state $h$ measurement: HE method

Table 1: Summary of steady-state $h$-coefficient measurements

\begin{tabular}{cc}
\hline Method & $h\left(\mathrm{~W} \cdot \mathrm{m}^{-2} \cdot \mathrm{K}^{-1}\right)$ \\
\hline \hline DM1 & $7.27 \pm 0.41$ \\
\hline DM2 & not applicable \\
\hline OT1 & $7.48 \pm 0.45$ \\
\hline OT2 & $7.46 \pm 0.45$ \\
\hline HE & $7.31 \pm 0.44$ \\
\hline
\end{tabular}

Basically, every method predicts a similar $h$-coefficient, close to the $7.7 \mathrm{~W} \cdot \mathrm{m}^{-2} . \mathrm{K}^{-1}$ standard value from standards ISO 14683 [30] and ISO 6946 [8]. The maximum discrepancy is around $0.2 \mathrm{~W} \cdot \mathrm{m}^{-2} \cdot \mathrm{K}^{-1}$, which is lower than the uncertainty of each method separately. This calculated uncertainty is almost independent of the method used. This is because the type-A uncertainty is here almost negligible with respect to the type-B uncertainty (measurements are issued from averages over a large amount of points).

It may be observed that the OT1 and OT2 methods lead to very close estimates of $h$. This confirms that the two ways of measuring the operative temperature $T_{\mathrm{op}}$ are equivalent in steady-state conditions. Indeed, the voltage applied to the thermoelectric coolers in the OT2 methods was almost null throughout the experiment. Therefore, the active compensation was not needed to maintain the surface heat flux equal to zero and the environment temperature sensor from ISO 9869-2 standard [6] is sufficient.

\section{Transient results}

The electrical heaters are here turned on for $5 \mathrm{~h}$ and then turned off. Figure 13 plots the air temperature evolution. The air temperature rises by about $8 \mathrm{~K}$. It is important to point out at this stage that the measurement noise is much more important when the heaters were turned on, especially for heat flux measurements. This noise is less due to the acquisition hardware that the air turbulence induced by the redictric fan heaters. The following paragraphs presents results for each $h$-measurement method.

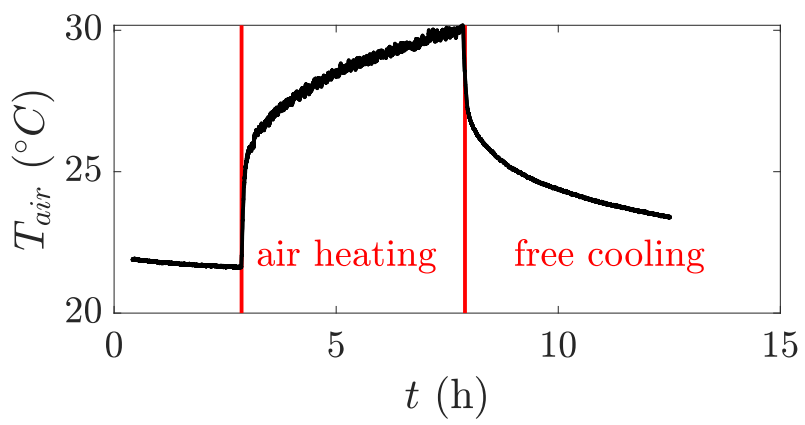

Figure 13: Evolution of the air temperature, measured $15 \mathrm{~cm}$ away from the wall

\subsection{DM1 method}

The heat fluxes and temperatures measured on surfaces A and B are shown in Fig. 14. Thanks to the heating resistance, temperature $T_{B}$ is always greater than $T_{A}$. In addition, $\varphi_{A}>$ $\varphi_{B}$ given the chosen convention (a positive heat flux enters the wall). The air heating and free-cooling phases are clearly visible in the measurements (between hour 3 and hour 8 and after hour 8, respectively).
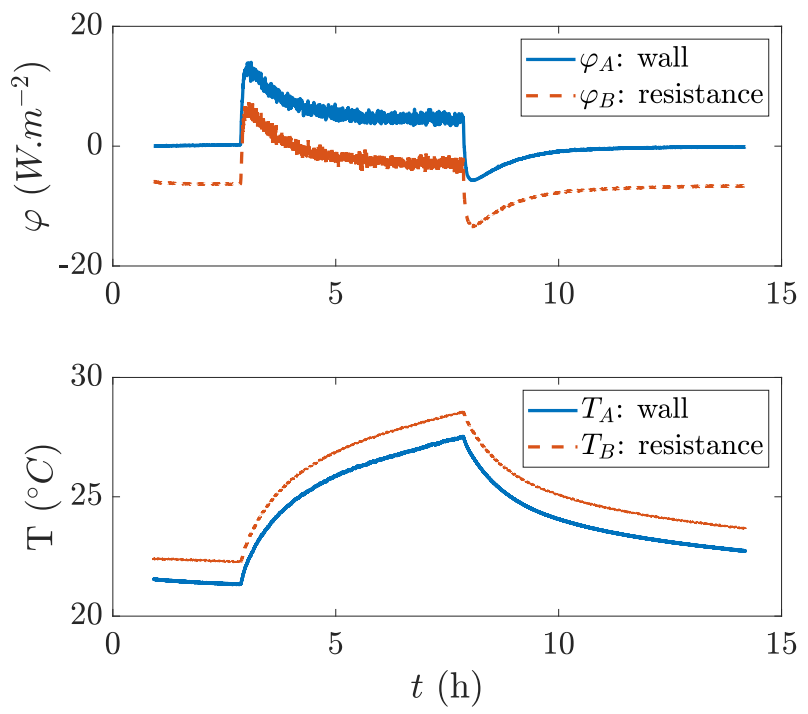

Figure 14: DM1 method: Surface heat flux and temperature measurements 
Figure 15 plots the calculated $h$-values (see Eq 9) as a function of time. Each point is obtained from temperatures and heat fluxes averaged over $5 \mathrm{~min}$. Because of the very high noise level on heat fluxes during the heating period, the uncertainties are larger than during the free-cooling phase. Surprisingly, the measured $h$-value is also smaller, especially just after the heaters are turned on. This observation might be explained by the sign of heat flux $\varphi_{\mathrm{B}}$ : it is negative before the heating phase, becomes positive at the beginning of it and comes back negative about an hour later. These heat flux inversions could alter the results as convective heat transfers may be different when the surface is either heated of cooled by the air.

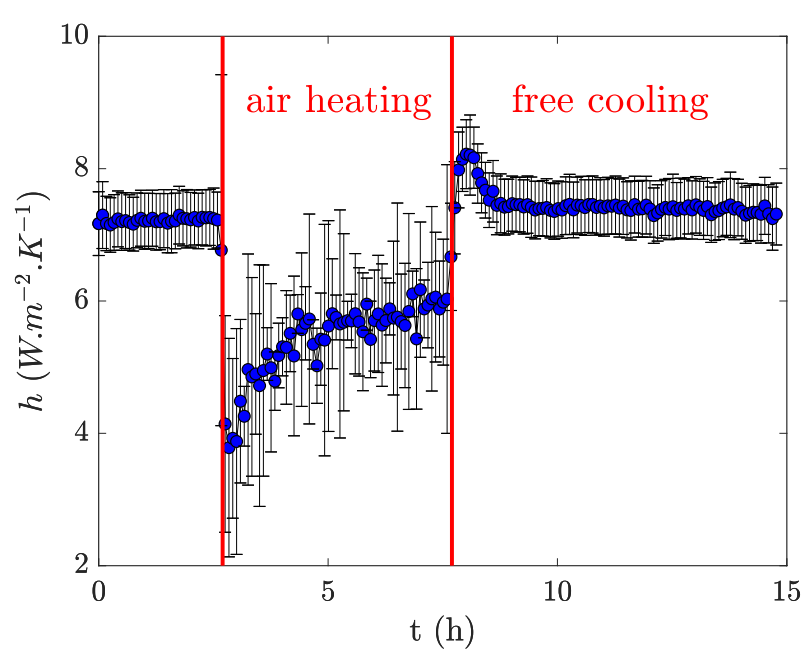

Figure 15: DM1 method: calculated $h$-coefficient against time

\subsection{DM2 method}

Similarly, measured heat fluxes and temperatures on surfaces $\mathrm{A}$ and $\mathrm{C}$ are given in Fig. 16. Because the wall and the piece of polystyrene have a different effusivity, they also have a different thermal response when heated by the air. Indeed, the insulated material heats up and cools down faster than the wall. The heat flux that enters it is consequently smaller.
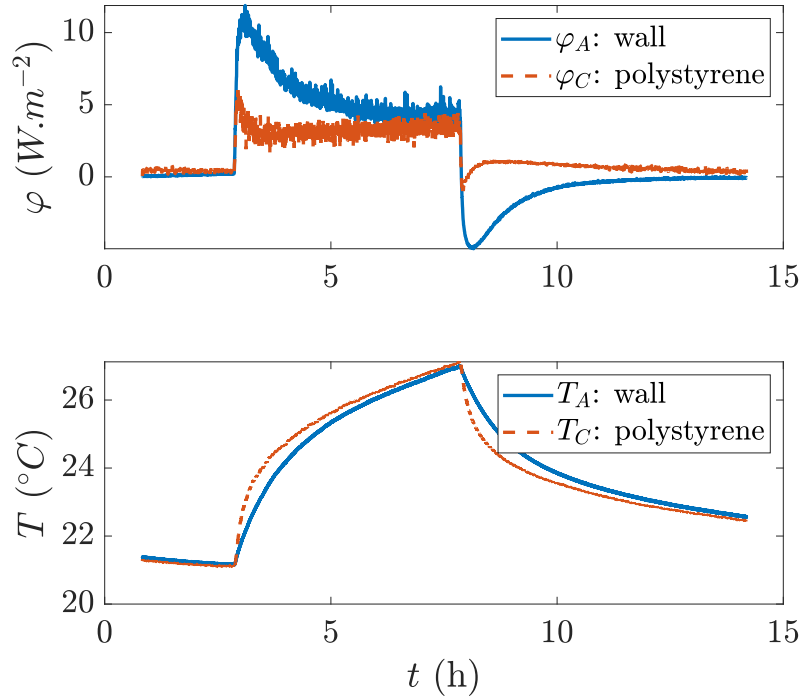

Figure 16: DM2 method: surface heat flux and temperature measurements

As shown in Figures 17 and 18, plotting the heat flux difference $\Delta \varphi$ against the temperature difference $\Delta T$ during each phase gives a straight line of slope $h$. This confirms that $h$ may be considered constant during each period. It is also greater during the first one, unlike predictions from the DM1 method. As already pointed out, there is much more noise when the heaters are on than off. It may also be observed that the density of points is not uniform: the smaller the $\Delta T$, the higher the density. Therefore, a classic linear regression would favor low $\Delta T$ measurements and would not necessarily be aligned with the few high $\Delta T$ points. This issue was fixed by applying weights to the points. These weights are inversely proportional to the point density (estimated by evenly discretizing the $\Delta T$ axis and counting the number of points present in each section).

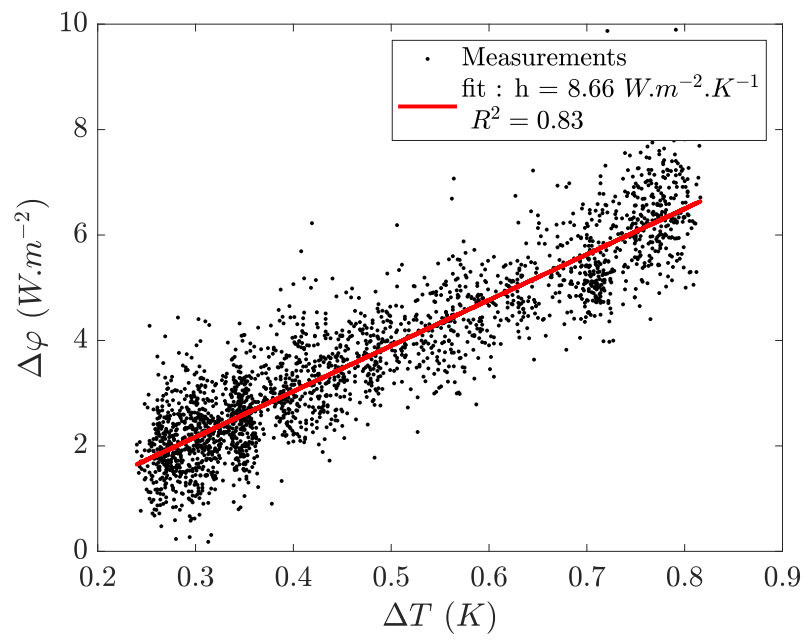

Figure 17: DM2 method: linear regression during the air heating period 


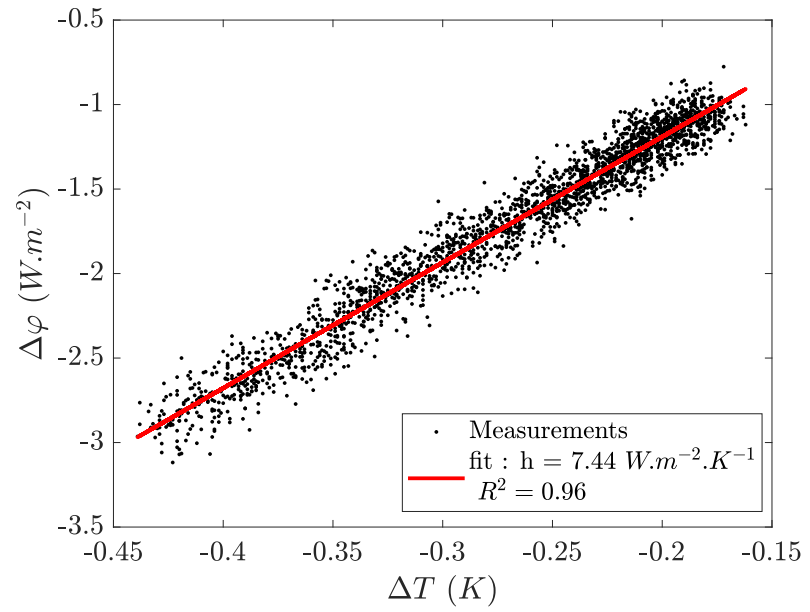

Figure 18: DM2 method: linear regression during the air free-cooling period

\subsection{OT1 method}

This method was not able to measure the $h$-coefficient in transient regime. Indeed, device $\mathrm{C}$ needs to be at thermal equilibrium to perform a proper measurement of the operative temperature $T_{\mathrm{op}}$. With a biased measurement of $T_{\mathrm{op}}$, the application of Eq. 12 leads to highly non-physical results. The different measured $T_{\text {op }}$ are presented in more details in the following paragraph (see Fig 20).

\subsection{OT2 method}

The first graph in Fig. 19 plots the heat fluxes $\varphi_{A}$ and $\varphi_{E}$ measured on the wall and on the device E.
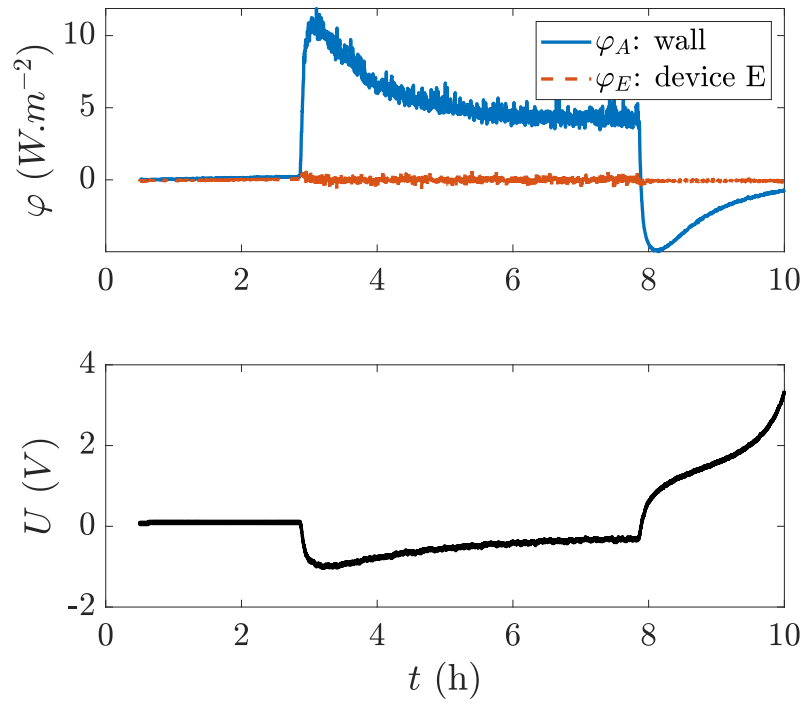

Figure 19: OT2 method: measured surface heat fluxes and TEC voltage command

As desired, $\varphi_{E}$ remains null during the experiment even though the room air is heated. The second graph displays the evolution of the voltage $U$ applied to the thermoelectric coolers enclosed inside the device. In the chosen convention, a negative voltage corresponds to a heating of the front surface. At the beginning of the experiment, the room is globally at thermal equilibrium (pseudo steady-state), hence $U \approx 0 \mathrm{~V}$. When the air temperature suddenly increases, the surface temperature of the zero-flux device has to increase as well in order to ensure $\varphi_{E}=0$, the TECs feeding voltage is negative. During the free-cooling period, the opposite trend is observed: the device front surface has to be cooled down to follow the decrease in the air temperature so $U>0$. The rear face of the device is therefore heated. Unfortunately, because it is directly fixed on the wall, the heat generated by the TECs cannot be rapidly dissipated. The system then diverges as $U$ has to be constantly increased to compensate for the rear face temperature elevation. As shown in Fig. 19, between the $9^{\text {th }}$ and $10^{\text {th }}$ hour of experiment, the voltage sharply increases until it reaches the safety limit set to $3 \mathrm{~V}$. Consequently, the design of the zero-flux device should be modified in order to dissipate the heat generated on its rear side and enable longer measurements of $T_{\mathrm{op}}$ during the free-cooling phase. This is beyond the scope of the present paper.

Figure 20 displays the measured temperatures. The air temperature $T_{\text {air }}$ measured with a thermocouple near the device is also plotted. These results are shown to illustrate the fact that the usual assumption $T_{\mathrm{op}}=T_{\mathrm{air}}$ in indoor building conditions is absolutely false in highly transient conditions like the present one. For instance, during the heating period, $T_{\mathrm{MRT}}$ is significantly lower than $T_{\text {air }}$ because the surface temperature of the surrounding objects is smaller thanks to their inertia which reduces $T_{\mathrm{op}}$.

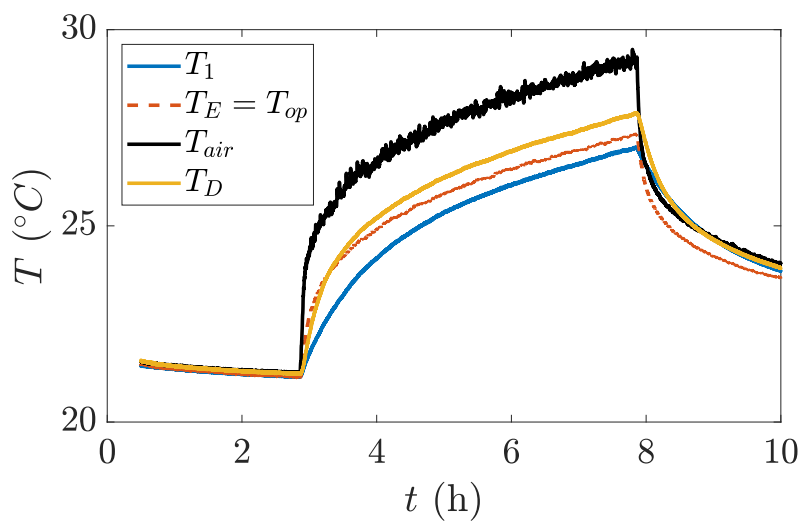

Figure 20: OT2 method: comparison of measured temperatures

Similarly to the DM2 method, the wall heat flux $\varphi_{A}$ is plotted against the temperature difference $\Delta T=T_{A}-T_{E}$ : see Figures 21 and 22. Despite the measurement noise (especially during the heating period), a straight line can again be fitted on the experiment to retrieve the $h$-coefficient value. Again, according to this method, $h$ is slightly greater during the first phase. 


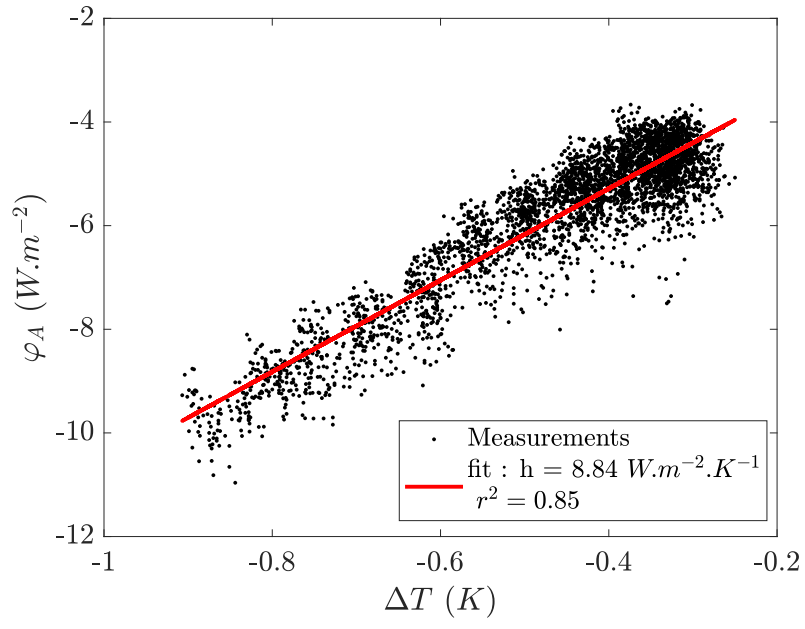

Figure 21: OT2 method: linear regression during the air heating period

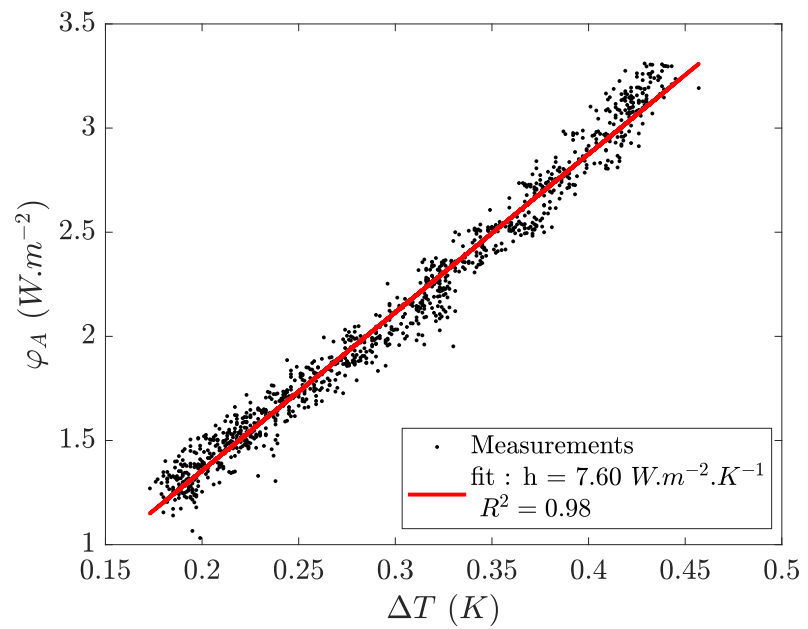

Figure 22: OT2 method: linear regression during the air free-cooling period

\subsection{HE method}

The heat flux and temperature measured on the Harmonic device (Device E) are plotted over a few oscillation periods of the heating phase in Fig. 23, and of the cooling phase in Fig. 24. As expected, the signal to noise ratio is better on signals in Fig. 24, especially for the heat flux.

The measured $h$ coefficients are plotted in Fig. 25 along with error bars. Each point corresponds to an analysis over 6 consecutive periods. With this method, the measured $h$ coefficient is higher during the heating period than during the free-cooling one. At the very beginning of each phase, there is a sharp increase or decrease of the calculated $h$-value. This result is not physical. It is due to a fast modification of the operative temperature : among the 6 consecutive periods used for the analysis, the first ones belong to a regime different from the last ones. Thus, for the measurement to be accurate, one should pay attention to avoid fast varying conditions.

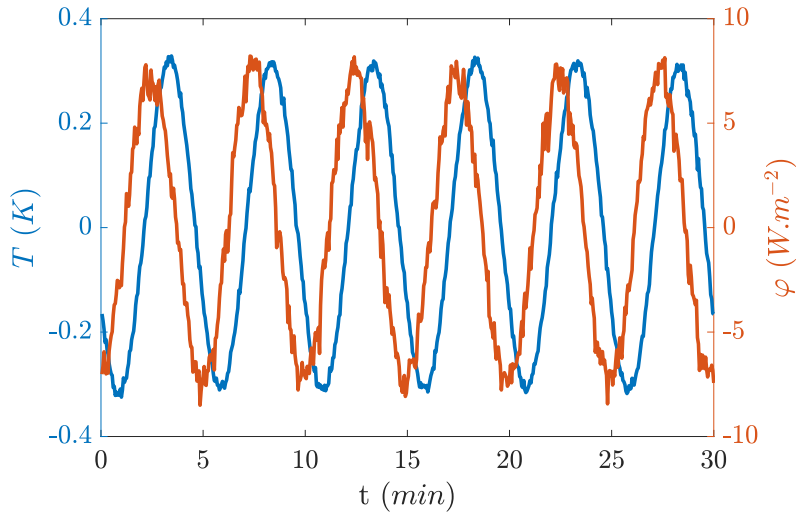

Figure 23: HE method: sample of heat flux and temperature measured on the device during the heating phase

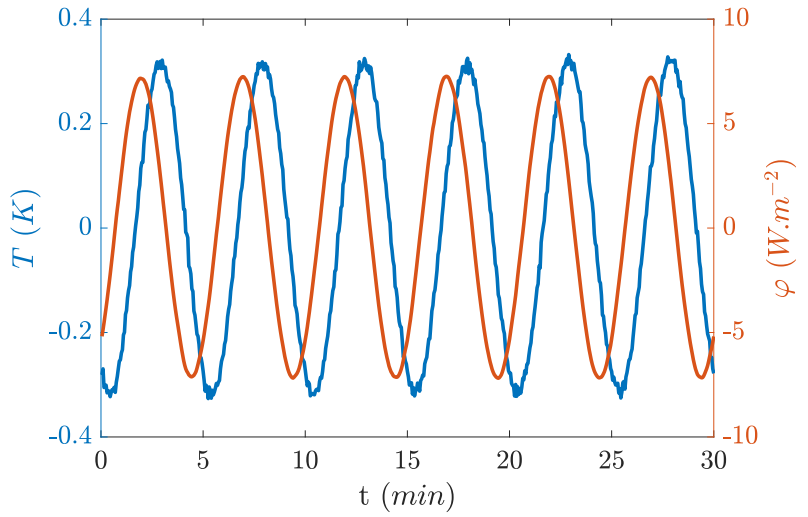

Figure 24: HE method: sample of heat flux and temperature measured on the device during the free-cooling phase

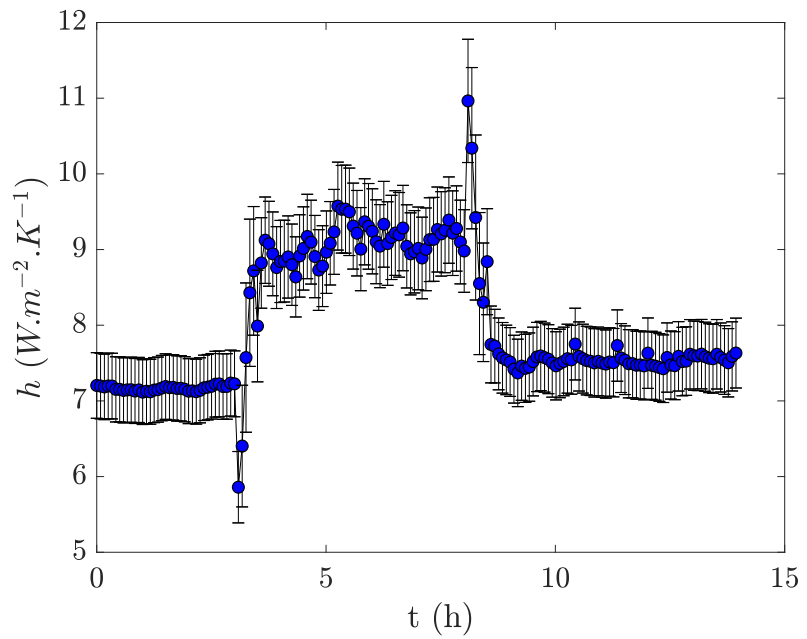

Figure 25: HE method: calculated $h$-coefficient value against time 


\section{Discussion}

\subsection{Comparison of methods}

To test the methods repeatability, the same experiment was repeated three times. The electrical heaters were turned on for 5 hours. The resulting measured $h$-coefficients are plotted in Figure 26 for both the heating and the cooling period. For the sake of brevity, only one $h$ value per period is retained for each method (the mean value over the period). No result from the OT1 method is provided since it proved to be only applicable in steady-state.

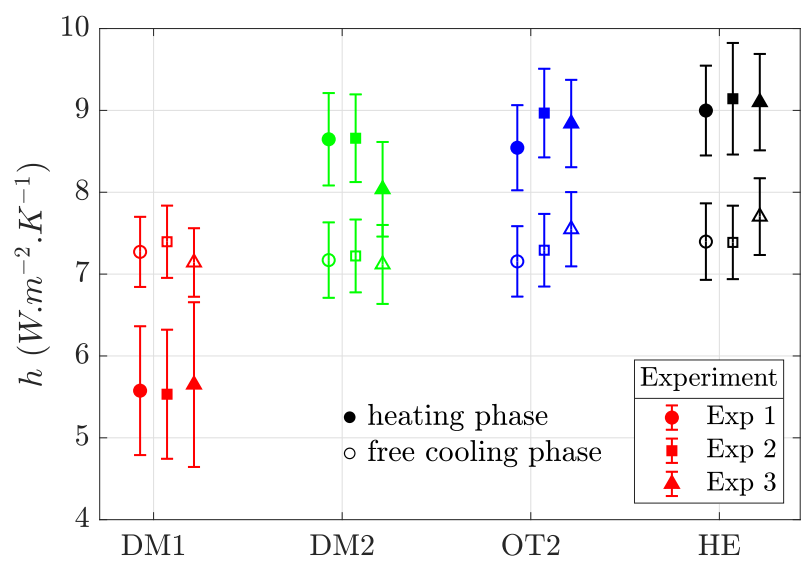

Figure 26: Comparison of $h$ measurement methods: heating and free-cooling periods (colors correspond to methods)

First, the measurement spread is globally more important during the heating phase. This is because of the higher noise on the heat flux measurements. Moreover, the uncertainties for the DM1 method are noticeably higher (about twice as big) when the heaters are on. Moreover, the uncertainties given by the other methods are not significantly affected by the noise level. This is explained by the fact that, in the current configuration, the type-A uncertainty is very small (typically $0.2 \mathrm{~W} \cdot \mathrm{m}^{-2} \cdot \mathrm{K}^{-1}$ ) compared to the type-B one (typically $0.45 \mathrm{~W} . \mathrm{m}^{-2} \cdot \mathrm{K}^{-1}$ ) while only type-A uncertainty takes the noise into account. The linear fits of the DM2 and OT2 methods are based on a high number of points (around 2000 in general) which reduces the statistical uncertainty given by Eq 20. In addition, with the HE method, the amplitude of the modulated heat flux is higher than other heat flux measured. The signal to noise ratio is therefore much higher, even during the heating period.

As seen in Fig. 26, all methods lead to similar results during the free-cooling period. Interestingly, the measured $h$-coefficients are close to the $7.7 \mathrm{~W} \cdot \mathrm{m}^{-2} \cdot \mathrm{K}^{-1}$ default value given by ISO standard 14683 [30] for indoor conditions. This result was to be expected because the free-cooling phase is close to standard heat exchange conditions encountered in buildings. In this particular configuration, using the standard value or the result of a measurement makes little difference. However, the proposed approach has the advantage of quantifying the uncertainty, while the standard value is given without confidence interval.

The average measured $h$-coefficient of the heating period is higher, around $8.5 \mathrm{~W} \cdot \mathrm{m}^{-2} \cdot \mathrm{K}^{-1}$ for the DM2 and OT2 methods and even higher with the HE one: around $9 \mathrm{~W} \cdot \mathrm{m}^{-2} \cdot \mathrm{K}^{-1}$. The DM1 method differs from the other ones as it predicts a lower $h$-value: around $5.5 \mathrm{~W} \cdot \mathrm{m}^{-2} \cdot \mathrm{K}^{-1}$.

As a conclusion, the DM1 and OT1 methods are not able to measure the $h$-coefficient in transient regimes in which the air is heated. The HE methods leads to a small overestimation during the heating period.

\subsection{Heat flux reconstruction}

As explained in the introduction, the measurement of the global $h$ coefficient enables to extrapolated the heat flux measured on a zone 1 to a nearby zone (see Eq 4). Heat fluxes are measured with $100 \times 100 \mathrm{~mm}^{2}$ HFM. An example of a heat flux reconstruction is shown in Fig. 27. In this example, the $h$ value measured by the DM2 method was used. The $h$ coefficient is considered constant within each period (air heating and free cooling). The measured and reconstructed heat fluxes $\varphi_{2}$ and $\varphi_{2}^{\prime}$ are rather well superimposed. The residuals, that is to say the difference $\varphi_{2}-\varphi_{2}^{\prime}$, are plotted in the second graph of Fig. 27. They are rather well centered around zero and almost not signed. A little bias is observed at the beginning of the free-cooling period. This is induced by the simplified approach of considering $h$ constant.

As a consequence, it is possible to reconstruct the measured heat flux on a zone 2 from contact measurements on a zone 1 , h-coefficient value and temperature difference between zones 1 and 2 .
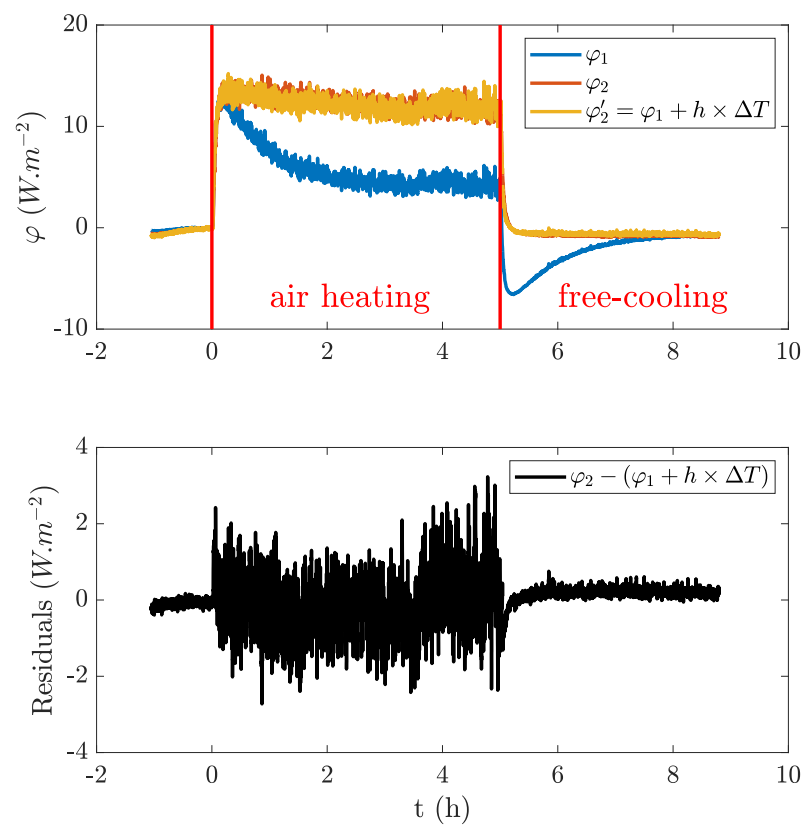

Figure 27: Heat flux extrapolation using measured $h$ coefficient

\section{Conclusion}

The measurement of heat losses on building walls is not trivial. First, heat flux meters cannot be used on thermal bridges because of the 2D or 3D nature of the heat transfers encountered. Second, the heat losses are rarely uniform over a wall 
and it is usually not convenient to cover the wall with contact sensors. At this time, there is no universal measurement methods allowing this quantification in all situations. Therefore, we proposed a new method consisting in extrapolating the local heat flux measured by a HFM on one location to the rest of the wall, even in the presence of thermal bridges. This requires the knowledge of the total heat exchange coefficient $h$. It is usually supposed known or estimated by empirical correlations from the literature. The accuracy of this approach is limited, especially because it cannot take into account the specificity of the encountered in situ configuration. In addition, in the context of a building, the choice of the reference temperatures to use in the correlations is not straightforward. This paper focuses on the indoor in situ measurement of $h$ in unsteady conditions. To this end, five $h$-measurement techniques were implemented: three of them are original whereas the other two are inspired from the published literature. The DM1 method rely on the measurements of both the temperature and the heat flux on two nearby surfaces: the naked wall and a heating resistance fixed on it. The DM2 approach is very similar, only the resistance is swapped with a piece of extruded polystyrene. The OT1 and OT2 methods are based on the measurement of the operative temperature, coupled with a heat flux measurement on the wall. While the OT1 method uses a passive device to measure $T_{\mathrm{op}}$, the OT2 one is based on an active compensation of surface heat losses thanks to thermoelectric coolers. Finally, in the HE approach, the temperature of a surface is modulated (using thermoelectric coolers as well) and a frequentist analysis is performed. These five techniques are not all applicable both in steady and transient states. Table 2 summarizes their applicability.

Table 2: Applicability of $h$-measurement methods

\begin{tabular}{|c|c|c|c|}
\hline \multirow[b]{2}{*}{ Method } & \multicolumn{2}{|r|}{ Applicable in : } & \multirow[b]{2}{*}{ Reference } \\
\hline & $\begin{array}{l}\text { Steady- } \\
\text { state }\end{array}$ & $\begin{array}{c}\text { Transient } \\
\text { state }\end{array}$ & \\
\hline DM1 & Yes & $\begin{array}{c}\text { Yes } \\
\text { but under-estimation }\end{array}$ & $\begin{array}{l}\text { inspired } \\
\text { from [15] }\end{array}$ \\
\hline DM2 & No & Yes & this paper \\
\hline OT1 & Yes & No & ISO 9869-2 \\
\hline $\mathrm{OT} 2$ & Yes & Yes & this paper \\
\hline $\mathrm{HE}$ & Yes & Yes & this paper \\
\hline
\end{tabular}

In steady-state, the different methods measured a very similar $h$ coefficient that proved to be close to the $7.7 \mathrm{~W} \cdot \mathrm{m}^{-2} \cdot \mathrm{K}^{-1}$ standard value from ISO 6946 [8]. To complete these results, an unsteady regime was artificially generated by heating the air of the room thanks to electric fan heaters. The unsteadyresults spread was more important than steady-state ones but most of the implemented methods measured higher $h$ coefficient during the heating phase (between 8.5 and $9 \mathrm{~W} \cdot \mathrm{m}^{-2} \cdot \mathrm{K}^{-1}$ ) than during the free-cooling one (around $7.5 \mathrm{~W} . \mathrm{m}^{-2} . \mathrm{K}^{-1}$, like in steady-state).

Future work will focus on the application of theses methods on outdoor building wall surfaces. Indeed, the outdoor $h$-coefficient is highly dependent on the wind direction and velocity. Therefore, rough estimations of it are very inaccurate so a direct measurement of $h$ exchange coefficient would be a relevant alternative.

Finally, it was demonstrated that it is possible to estimate heat losses onto a wall surface if the heat flux is measured locally in a given "reference" zone and if the global heat exchange coefficient is measured using one of the proposed methods. The third information required is the temperature difference between the reference zone and the rest of the wall surface. We have tested successfully this approach by measuring the temperature difference between two distinct zones with a thermocouple. The use of an infrared camera should allow to obtain accurately a map of temperature difference on the wall surface and thus to obtain a map of heat losses. This method has now to be tested in several building walls for its final validation.

\section{References}

[1] D. Johnston, D. Miles-Shenton, and D. Farmer. Quantifying the domestic building fabric a 'performance gap'. Building Services Engineering Research and Technology, 36(5):614-27, 2015.

[2] David Bienvenido-Huertas, Juan Moyano, David Marín, and Rafael Fresco-Contreras. Review of in situ methods for assessing the thermal transmittance of walls. Renewable and Sustainable Energy Reviews, 102:356-371, 2019.

[3] Johann Meulemans, Florent Alzetto, David Farmer, and Christopher Gorse. Qub/e: A novel transient experimental method for in situ measurements of the thermal performance of building fabrics. In Building Information Modelling, Building Performance, Design and Smart Construction, pages 115-127. Springer, 2017.

[4] Johann Meulemans. An assessment of the qub/e method for fast in situ measurements of the thermal performance of building fabrics in cold climates. In Cold Climate HVAC Conference, pages 317-326. Springer, 2018.

[5] ISO 9869-1:2014. Thermal insulation - Building elements - In-situ measurement of thermal resistance and thermal transmittance - Part 1: Heat flow meter method. International Standards Organisation, 2014.

[6] ISO 9869-2:2018. Thermal insulation - Building elements - In-situ measurement of thermal resistance and thermal transmittance - Part 2: Infrared method for frame structure dwelling. International Standards Organisation, 2018.

[7] A. François, L. Ibos, V. Feuillet, and J. Meulemans. Building thermal bridge heat losses quantification by infrared thermography. Steady-state evaluation and uncertainty calculation. In Journal of Physics: Conference Series, volume 1343, page 012171. IOP Publishing, 2019.

[8] ISO 6946:2017. Building components and building elements - Thermal resistance and thermal transmittance - Calculation methods. International Standards Organisation, 2017. 
[9] A. Bejan. Convection heat transfer. John wiley \& sons, 2013.

[10] H.B. Awbi and A. Hatton. Natural convection from heated room surfaces. Energy and buildings, 30(3):233244, 1999.

[11] E. Dascalaki, M. Santamouris, C.A. Balaras, and D.N. Asimakopoulos. Natural convection heat transfer coefficients from vertical and horizontal surfaces for building applications. Energy and Buildings, 20(3):243-249, 1994.

[12] S.E.G. Jayamaha, N.E. Wijeysundera, and S.K. Chou. Measurement of the heat transfer coefficient for walls. Building and Environment, 31(5):399-407, 1996.

[13] A. Hagishima and J. Tanimoto. Field measurements for estimating the convective heat transfer coefficient at building surfaces. Building and Environment, 38(7):873881, 2003.

[14] D.L. Loveday and A.H. Taki. Convective heat transfer coefficients at a plane surface on a full-scale building facade. International Journal of Heat and Mass Transfer, 39(8):1729-1742, 1996.

[15] N. Ito. Field experiment study on the convective heat transfer coefficient on exterior surface of a building. ASHRAE Trans.;(United States), 78, 1972.

[16] K.E.A. Ohlsson, R. Östin, and T. Olofsson. Accurate and robust measurement of the external convective heat transfer coefficient based on error analysis. Energy and Buildings, 117:83-90, 2016.

[17] K.E.A. Ohlsson, R. Östin, S. Grundberg, and T. Olofsson. Dynamic model for measurement of convective heat transfer coefficient at external building surfaces. Journal of Building Engineering, 7:239-245, 2016.

[18] A.J.N. Khalifa and R.H. Marshall. Validation of heat transfer coefficients on interior building surfaces using a real-sized indoor test cell. International Journal of Heat and Mass Transfer, 33(10):2219-2236, 1990.

[19] P. Wallentén. Convective heat transfer coefficients in a full-scale room with and without furniture. Building and Environment, 36(6):743-751, 2001.

[20] E. Mayer. Measurement of the convective surface coefficient of heat transfer of man. Room Vent, 87, 1987.

[21] S.R. Delaforce, E.R. Hitchin, and D.M.T. Watson. Convective heat transfer at internal surfaces. Building and Environment, 28(2):211-220, 1993.

[22] A.D. Irving, T. Dewson, G. Hong, and B. Day. Time series estimation of convective heat transfer coefficients. Building and Environment, 29(1):89-96, 1994.

[23] B. Griffith, D. Turler, H. Goudey, and D. Arasteh. Experimental techniques for measuring temperature and velocity fields to improve the use and validation of building heat transfer models. Technical report, Lawrence Berkeley National Lab.(LBNL), Berkeley, CA (United States), 1998.
[24] M. Davies, C. Martin, M. Watson, and C.N. Riain. The development of an accurate tool to determine convective heat transfer coefficients in real buildings. Energy and Buildings, 37(2):141-145, 2005.

[25] S. Kato, K. Kuroki, and S. Hagihara. Method of in-situ measurement of thermal insulation performance of building elements using infrared camera. In 6th International Conference on Indoor Air Quality, Ventilation 85 Energy Conservation in Buildings-IAQVEC, 2007.

[26] H. Preston-Thomas. The international temperature scale of 1990 (its-90). metrologia, 27(1):3, 1990.

[27] IEC BIPM, ILAC IFCC, and IUPAC ISO. Iupap and oiml 2008 guide to the expression of uncertainty in measurement (gum: 1995 with minor corrections) bureau international des poids et mesures.

[28] T. Metzger and D. Maillet. Multisignal least squares: Dispersion, bias, and regularization. In Thermal Measurements and Inverse Techniques, pages 613-632. CRC Press, 2011.

[29] National Instruments. Note d'application 043. Technical report.

[30] ISO 14683:2017. Thermal bridges in building construction - Linear thermal transmittance - Simplified methods and default values. International Standards Organisation, 2017.

\section{A Appendix: type-A uncertainty calculation of the HE method}

\section{A.1 Amplitude and phase uncertainty of a Fourier transform}

The Fourier transform of a N-point sequence $x$ (temperature measurement with time for instance) is given by:

$$
F T(k)=\frac{1}{N} \sum_{n=0}^{N-1} x(n) \exp \left(-j k \beta_{n}\right)
$$

with $\beta_{n}=2 \pi n / N$. The real and imaginary parts of $X$ are therefore:

$$
\left\{\begin{array}{l}
R(k)=\frac{1}{N} \sum_{n=0}^{N-1} x(n) \cos \left(k \beta_{n}\right) \\
I(k)=\frac{1}{N} \sum_{n=0}^{N-1} x(n) \sin \left(k \beta_{n}\right)
\end{array}\right.
$$

The amplitude $A$ and phase $\zeta$ of the $k$-th harmonic are as follows:

$$
\left\{\begin{array}{l}
A(k)=2 * \sqrt{R(k)^{2}+I(k)^{2}} \\
\zeta(k)=\arctan \left(-\frac{I(k)}{R(k)}\right)
\end{array}\right.
$$

Let assume that each point of the $x$ sequence is affected by an uncertainty $u_{x}$ (measurement noise). The uncertainty is propagated to $R$ and $I$ :

$$
\left\{\begin{array}{l}
u_{R(k)}^{2}=\sum_{n=0}^{N-1}\left(\frac{\partial R(k)}{\partial x(n)}\right)^{2} u_{x(n)}^{2} \\
u_{I(k)}^{2}=\sum_{n=0}^{N-1}\left(\frac{\partial I(k)}{\partial x(n)}\right)^{2} u_{x(n)}^{2}
\end{array}\right.
$$


leading to:

$$
\left\{\begin{array}{l}
u_{R(k)}^{2}=\frac{1}{N^{2}} \sum_{n=0}^{N-1} \cos ^{2}\left(k \beta_{n}\right) u_{x}^{2} \\
u_{I(k)}^{2}=\frac{1}{N^{2}} \sum_{n=0}^{N-1} \sin ^{2}\left(k \beta_{n}\right) u_{x}^{2}
\end{array}\right.
$$

For $k \neq 0$, it comes:

$$
u_{R(k)}^{2}=u_{I(k)}^{2}=\frac{u_{x}^{2}}{2 N}
$$

Similarly, the uncertainty are propagated to the amplitude $A$ and phase $\zeta$ :

$$
\left\{\begin{array}{l}
u_{A(k)}^{2}=\frac{2 u_{x}^{2}}{N} \\
u_{\zeta(k)}^{2}=\frac{2 u_{x}^{2}}{N A^{2}(k)}
\end{array}\right.
$$

\section{A.2 $h$ calculation}

The $h$ value is obtained from the ratio of the heat flux and temperature Fourier transform at the excitation frequency $f$ (associated to the index $k_{f}$ ):

$$
h=\Re\left(\frac{F T_{\varphi}\left(k_{f}\right)}{F T_{\mathrm{T}}\left(k_{f}\right)}\right)
$$

Let $F T_{\varphi}\left(k_{f}\right)=R_{\varphi}+j I_{\varphi}$ and $F T_{\mathrm{T}}\left(k_{f}\right)=R_{\mathrm{T}}+j I_{T}$. The previous equation becomes:

$$
h=\frac{R_{\varphi} R_{\mathrm{T}}+I_{\varphi} I_{\mathrm{T}}}{R_{\mathrm{T}}^{2}+I_{\mathrm{T}}^{2}}=\frac{\mathrm{Num}}{\text { Den }}
$$

With Num $=R_{\varphi} R_{\mathrm{T}}+I_{\varphi} I_{\mathrm{T}}$ and Den $=R_{\mathrm{T}}^{2}+I_{\mathrm{T}}^{2}$, the four partial derivatives of $h$ are given by:

$$
\begin{aligned}
\frac{\partial h}{\partial R_{\varphi}} & =\frac{R_{\mathrm{T}}}{\operatorname{Den}} \\
\frac{\partial h}{\partial I_{\varphi}} & =\frac{I_{\mathrm{T}}}{\operatorname{Den}} \\
\frac{\partial h}{\partial R_{\mathrm{T}}} & =\frac{R_{\varphi} \text { Den }-2 R_{\mathrm{T}} \mathrm{Num}}{\operatorname{Den}^{2}} \\
\frac{\partial h}{\partial I_{\mathrm{T}}} & =\frac{I_{\varphi} \text { Den }-2 I_{\mathrm{T}} \mathrm{Num}}{\text { Den }^{2}}
\end{aligned}
$$

The uncertainty of each of these parameters are given by Eq. 30. Once again, the uncertainty propagation law is applied to derive the $h$ measurement uncertainty $u_{h}$. After simplification:

$$
u_{\mathrm{h}}=\sqrt{\frac{2}{N}} \frac{1}{A_{\mathrm{T}}} \sqrt{u_{\varphi}^{2}+\left(\frac{A_{\varphi}}{A_{\mathrm{T}}}\right)^{2} u_{\mathrm{T}}^{2}}
$$

with $A_{\mathrm{T}}$ and $A_{\varphi}$ the temperature and heat flux amplitudes (see Eq. 27). For given noise levels $u_{\varphi}$ and $u_{\mathrm{T}}$, the uncertainty on $h$ decreases in $1 / \sqrt{N}$ with $N$.

This uncertainty may also be expressed in terms of the uncertainty over the amplitudes $u_{A_{\varphi}}$ and $u_{A_{T}}$ (see Eq. 31):

$$
u_{h}=\frac{1}{A_{\mathrm{T}}} \sqrt{u_{A_{\varphi}}^{2}+\left(\frac{A_{\varphi}}{A_{\mathrm{T}}}\right)^{2} u_{A_{T}}^{2}}
$$

It is interesting to point out that $u_{h}$ from the previous equation is equal to the uncertainty of the amplitude ratio $A_{\varphi} / A_{\mathrm{T}}$. 